\title{
On the stability of fragments and thermalization achieved in neutron-rich heavy-ion collisions
}

\author{
Sukhjit Kaur ${ }^{1, a}$ \\ ${ }^{1}$ Department of Physics, Panjab University, Chandigarh -160 014, INDIA
}

\begin{abstract}
Using the isospin-dependent quantum molecular dynamics model, we study the stability of the fragments and the thermalization achieved in the collision of a neutronrich colliding pair at energies where the maximum production of intermediate mass fragments $\left(E_{c . m}^{\max }\right)$ occurs. In particular, we check the stability of fragments through the persistence coefficient and gain term and information about the thermalization and stopping is obtained via the relative momentum, anisotropy ratio, and rapidity distribution. Our findings show a weak dependence of these quantities on the neutron content of the colliding pair.
\end{abstract}

\section{Introduction}

Due to the interplay of correlations and fluctuations emerging in a collision, a highly excited nuclear system, formed in intermediate energy heavy-ion collisions, decays into several light and intermediate size fragments. The fragmentation pattern is found to be affected by the size of the colliding nuclei, the incident energy as well as the impact parameter [1-5]. In the literature several studies have been made to study the fragmentation pattern and thermalization achieved in heavy-ion reactions. Puri and co-workers [6] studied multifragmentation at the energy of vanishing flow in central heavy-ion collisions and found a power law dependence of different fragment multiplicities at the energy of vanishing flow. In an another study, the degree of stopping is found to decrease with the increase in impact parameter as well as at very high energies [3]. Also, heavier masses are found to equilibrate more than the lighter systems [4]. Sood and Kaur [5] studied various properties of fragments at $E_{c . m}^{\max }$. (the energy at which maximal production of intermediate mass fragments occurs) and found that for a complete stopping of incoming nuclei very heavy systems are required. The mass dependence of various quantities is found to follow a power law dependence.

With the availability of radioactive ion beams (RIBs), it has become possible to study neutronrich heavy-ion collisions. The neutron content of a colliding pair is found to affect the multifragment decay [7-14], collective as well as elliptic flow [15-17]. Recently, Puri and co-worker [12] studied the isospin effects on the energy of peak mass production. Both $E_{c . m}^{\max }$. (the energy at which maximal production of intermediate mass fragments occurs) and $\left\langle N_{\mathrm{IMF}}\right\rangle^{\max }$ (the maximum number of intermediate mass fragments) are found to be insensitive to the isospin dependence of the nucleon-nucleon cross section but $E_{c . m}^{\max }$. showed sensitivity towards the symmetry energy. One is, therefore, interested in understanding how nuclear dynamics behaves at this peak energy. A limited number of studies

\footnotetext{
ae-mail: sukhjtkaur@gmail.com
} 
have been made to check the role of the neutron content of a colliding pair on the nuclear dynamics. The role of the neutron content on the participant-spectator matter and thermalization achieved in a reaction is checked at the energy of vanishing flow and a weak dependence is obtained on the neutron content of the colliding systems [18]. No study was made to see the role of the neutron content of the colliding pair on the stability of the fragments and thermalization at $E_{c . m \text {. }}^{\max }$. We plan to study here the stability of the fragments and the degree of stopping reached in neutron-rich heavy-ion reactions at the peak center-of-mass energy.

\section{The Model}

The present study is made within the framework of the isospin-dependent quantum molecular dynamics (IQMD) model [19], which treats different charge states of nucleons, $\Delta$ 's, and pions explicitly. The IQMD and quantum molecular dynamics (QMD) models [20] are found to successfully explain the underlying physics of a large number of observables from low to relativistic energies $[4,19,21-$ 23]. In the IQMD model, successfully initialized nuclei are boosted towards each other with the proper center-of-mass velocity using relativistic kinematics. In this model, baryons are represented by Gaussian-shaped density distributions

$$
f_{i}(\vec{r}, \vec{p}, t)=\frac{1}{\pi^{3} \hbar^{3}} \exp \left(-\left[\vec{r}-\vec{r}_{i}(t)\right]^{2} \frac{1}{2 L}\right) \exp \left(-\left[\vec{p}-\vec{p}_{i}(t)\right]^{2} \frac{2 L}{\hbar^{2}}\right) .
$$

The centroids of the Gaussians in a nucleus are randomly distributed in a phase space sphere with $\mathrm{R}=1.12 \mathrm{~A}^{1 / 3} \mathrm{fm}$ corresponding to a ground state density of $\rho_{o}=0.17 \mathrm{fm}^{-3}$. Each nucleon occupies a volume of $h^{3}$, so that phase space is uniformly filled. The initial momenta of the nucleons are randomly chosen between 0 and the Fermi momentum $\left(\vec{p}_{F}\right)$. The Fermi momentum depends on the ground state density. For $\rho_{o}=0.17 \mathrm{fm}^{-3}$, it has a value of about $p_{F} \approx 268 \mathrm{MeV} / \mathrm{c}$. The nucleons of the target and projectile interact by two- and three-body Skyrme forces, a Yukawa potential, and Coulomb interactions. A symmetry potential between protons and neutrons corresponding to the Bethe-Weizsäcker mass formula has been included along with the explicit charge states of all baryons and mesons. The propagation is governed by the classical equations of motion:

$$
\frac{d \vec{r}_{i}}{d t}=\frac{d\langle H\rangle}{d \vec{p}_{i}}, \quad \frac{d \vec{p}_{i}}{d t}=-\frac{d\langle H\rangle}{d \vec{r}_{i}}
$$

where $\mathrm{H}$ stands for the Hamiltonian which is given by:

$$
\begin{array}{r}
\langle H\rangle=\langle T\rangle+\langle V\rangle \\
=\sum_{i} \frac{p_{i}^{2}}{2 m_{i}}+\sum_{i} \sum_{j>i} \int f_{i}(\vec{r}, \vec{p}, t) V^{i j} f_{j}\left(\overrightarrow{r^{\prime}}, \overrightarrow{p^{\prime}}, t\right) d \vec{r} d \overrightarrow{r^{\prime}} d \vec{p} d \overrightarrow{p^{\prime}} .
\end{array}
$$

The baryon potential $V^{i j}$, in the equation above reads as:

$$
\begin{aligned}
V^{i j}\left(\overrightarrow{r^{\prime}}-\vec{r}\right)= & V_{\text {Skyrme }}^{i j}+V_{\text {Yukawa }}^{i j}+V_{\text {Coul }}^{i j}+V_{\text {sym }}^{i j} \\
= & {\left[t_{1} \delta\left(\overrightarrow{r^{\prime}}-\vec{r}\right)+t_{2} \delta\left(\overrightarrow{r^{\prime}}-\vec{r}\right) \rho^{\gamma-1}\left(\frac{\overrightarrow{r^{\prime}}+\vec{r}}{2}\right)\right] } \\
& +t_{3} \frac{\exp \left(-\left|\left(\overrightarrow{r^{\prime}}-\vec{r}\right)\right| / \mu\right)}{\left(\left|\left(\overrightarrow{r^{\prime}}-\vec{r}\right)\right| / \mu\right)}+\frac{Z_{i} Z_{j} e^{2}}{\left|\left(\overrightarrow{r^{\prime}}-\vec{r}\right)\right|} \\
& +t_{4} \frac{1}{\varrho_{o}} T_{3 i} T_{3 j} \delta\left(\overrightarrow{r^{\prime}}-\vec{r}\right) .
\end{aligned}
$$


Here $t_{3}=-6.66 \mathrm{MeV}, \mu=0.4 \mathrm{fm}, t_{4}=4 \mathrm{C}$ with $\mathrm{C}=32 \mathrm{MeV}$ and $Z_{i}$ and $Z_{j}$ denote the charges of the $i t h$ and $j$ th baryon, and $T_{3 i}$ and $T_{3 j}$ are their respective $T_{3}$ components (i.e. $1 / 2$ for protons and $-1 / 2$ for neutrons). The parameters $\mu$ and $t_{1}, \ldots, t_{4}$ are adjusted to the real part of the nucleon optical potential. The standard Skyrme-type parametrization is employed for the density dependence of the nucleon optical potential. Two nucleons undergo a scattering if they are closer than a distance of $\sqrt{\frac{\sigma^{\text {tot }}(\sqrt{s})}{\pi}}$. Here $\sigma^{\text {tot }}(\sqrt{s})$ represents the total nucleon-nucleon cross section and ' $\sqrt{s}$ ' is the center-ofmass energy. This scattering is further subjected to the fulfillment of the Pauli principle. In the IQMD model, explicit Pauli blocking is included i.e., Pauli blocking of the neutrons and of the protons are treated separately. Any scattering that violates the Pauli principle is neglected. Whenever an attempted collision is blocked, the scattering partners maintain the original momenta prior to scattering.

The phase space generated with the IQMD model is stored at different time steps. One needs a clusterization algorithm to construct the fragments from the present phase space. For the present study, we use the minimum spanning tree (MST) method. In the MST method, two nucleons are allowed to share the same fragment if their centroids are closer than a distance $r_{\text {min }}$,

$$
\left|\mathbf{r}_{\mathbf{i}}-\mathbf{r}_{\mathbf{j}}\right| \leq r_{\min }
$$

where $\mathbf{r}_{\mathbf{i}}$ and $\mathbf{r}_{\mathbf{j}}$ are the spatial positions of both nucleons. The value of the clusterization radius is taken to be $2.8 \mathrm{fm}$.

\section{Results and discussion}

We simulated several thousands events of $\mathrm{Ca}+\mathrm{Ca}, \mathrm{Ni}+\mathrm{Ni}, \mathrm{Zr}+\mathrm{Zr}, \mathrm{Sn}+\mathrm{Sn}$ and $\mathrm{Xe}+\mathrm{Xe}$ reactions at the incident energies at which the maximal production of intermediate mass fragments (IMFs) occurs. In particular, we simulated the reactions of ${ }^{40} \mathrm{Ca}+{ }^{40} \mathrm{Ca},{ }^{56} \mathrm{Ni}+{ }^{56} \mathrm{Ni},{ }^{80} \mathrm{Zr}+{ }^{80} \mathrm{Zr},{ }^{100} \mathrm{Sn}+{ }^{100} \mathrm{Sn}$, ${ }^{110} \mathrm{Xe}+{ }^{110} \mathrm{Xe},{ }^{52} \mathrm{Ca}+{ }^{52} \mathrm{Ca},{ }^{72} \mathrm{Ni}+{ }^{72} \mathrm{Ni},{ }^{104} \mathrm{Zr}+{ }^{104} \mathrm{Zr},{ }^{129} \mathrm{Sn}+{ }^{129} \mathrm{Sn},{ }^{140} \mathrm{Xe}+{ }^{140} \mathrm{Xe},{ }^{60} \mathrm{Ca}+{ }^{60} \mathrm{Ca},{ }^{84} \mathrm{Ni}+{ }^{84} \mathrm{Ni}$, ${ }^{120} \mathrm{Zr}+{ }^{120} \mathrm{Zr},{ }^{150} \mathrm{Sn}+{ }^{150} \mathrm{Sn}$ and ${ }^{162} \mathrm{Xe}+{ }^{162} \mathrm{Xe}$ at an impact parameter of $\mathrm{b} / \mathrm{b}_{\max }=0.2-0.4$. We, here, use a soft equation of state along with the standard isospin- and energy-dependent cross section and reactions are followed till $300 \mathrm{fm} / \mathrm{c}$.

One of the important aspects in fragmentation is the stability of the fragments as well as the nucleons surrounding a fragment. The change in the nucleon content of the fragments between two successive time steps can be quantified with the help of the persistence coefficient $[24,25]$.

Let the number of pairs of nucleons in cluster $\mathrm{C}$ at time $t$ be $\chi_{C}(t)=0.5 * N_{C}\left(N_{C}-1\right)$. At the time $\Delta t$ later, it is possible that some of the nucleons belonging to cluster $C$ have left the cluster and are part of another cluster or are set free or others may have entered the cluster. Now, let $N_{C_{D}}$ be the number of nucleons that were in the cluster $\mathrm{C}$ at time $t$ and at $t+\Delta t$ are in cluster $\mathrm{D}$. We define

$$
\Phi_{C}(t+\Delta t)=\sum_{D} 0.5 * N_{C_{D}}\left(N_{C_{D}}-1\right)
$$

Here the sum runs over all fragments D present at time $t+\Delta t$.

The persistence coefficient of cluster $C$ can be defined as $[24,25]$ :

$$
P_{C}\left(t+\frac{\Delta t}{2}\right)=\Phi_{C}(t+\Delta t) / \chi_{C}(t)
$$

The persistence coefficient averaged over an ensemble of fragments is defined as:

$$
\left\langle P\left(t+\frac{\Delta t}{2}\right)\right\rangle=\frac{1}{N_{f_{t}}} \sum_{C} P_{C}\left(t+\frac{\Delta t}{2}\right),
$$


where $\mathrm{N}_{f_{t}}$ is the number of fragments present at time $t$ in a single simulation. The quantity is then averaged over a large number of IQMD simulations. The stability of a fragment between two consecutive time steps can be measured through the persistence coefficient. If the fragment does not emit a nucleon between two time steps, the persistence coefficient is one. On the other hand, if the fragment disintegrates completely, the persistence coefficient will be zero. If we remove one nucleon from a fragment $\mathrm{C}$, the persistence coefficient is $\mathrm{P}_{C}(t+\Delta t / 2)=\left(\mathrm{N}_{C}-2\right) / \mathrm{N}_{C}$ i.e., 0.333 for $\mathrm{N}_{C}=3$ and 0.8 for $\mathrm{N}_{C}=10$. For example for mass 10 , when one nucleon is emitted we have two entities at a later time step consisting of the free nucleon and the fragment with mass 9 . The $\mathrm{P}_{C}(t+\Delta t / 2)$ is the contribution from all such entities existing at later times. It, then, measures the tendency of the members of a given cluster to remain together.

In Fig. 1, we display the persistence coefficient for light charged particles (LCPs; $2 \leq \mathrm{A} \leq 4)$ (left panels) and intermediate mass fragments (IMFs; $5 \leq \mathrm{A} \leq A_{\text {tot }} / 6$ ) (right panels) for $\mathrm{N} / \mathrm{Z}=1.0,1.6$ and 2.0. The various lines are explained in the caption. It is clear from the figure that the saturation value of the persistence coefficient is slightly higher in case of LCPs as compared to IMFs. One can conclude that the final fragments are formed after $100 \mathrm{fm} / \mathrm{c}$. Before that time there is a strong exchange of

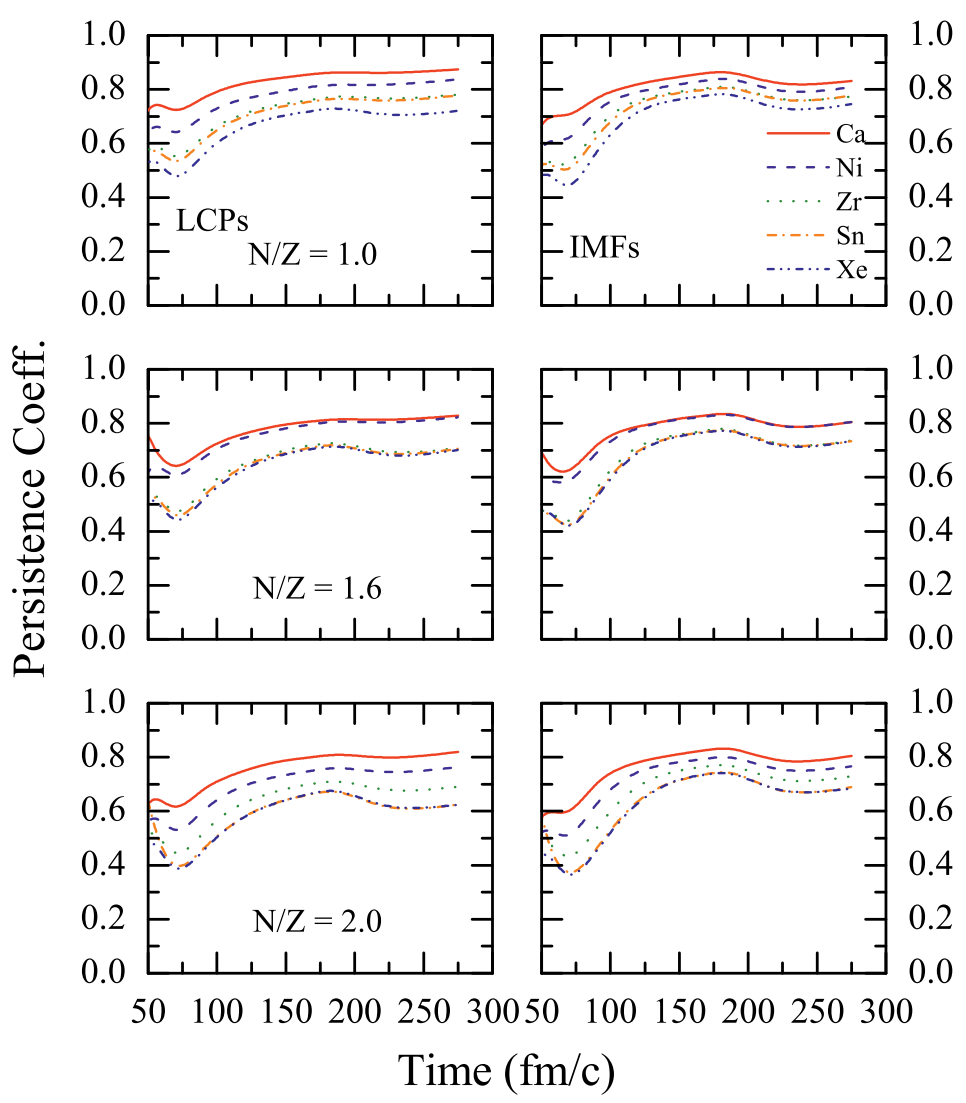

Figure 1. (Color Online) The persistence coefficient as a function of time for LCPs (left panels) and IMFs (right panels). Solid, dashed, dotted, dash-dotted and dash-double-dotted lines are for reactions of $\mathrm{Ca}+\mathrm{Ca}, \mathrm{Ni}+\mathrm{Ni}$, $\mathrm{Zr}+\mathrm{Zr}, \mathrm{Sn}+\mathrm{Sn}$ and $\mathrm{Xe}+\mathrm{Xe}$, respectively. 
nucleons between the fragments. The number of intermediate size fragments increases because the largest fragment falls finally into this mass bracket. The persistence coefficient reaches its asymptotic value later due to the interaction between fragments as well as between fragments and free nucleons. Due to this interaction nucleons are sometimes absorbed or emitted from the fragments. This process changes the details but not the general structure of the fragmentation pattern. The value of persistence coefficient is lower in the case of heavier colliding nuclei compared to lighter ones, which indicates the ongoing interactions among the nucleons. The persistence coefficient shows a weak dependence on the neutron content of colliding pair.

The persistence coefficient tells one about the stability of different fragments between two successive time steps. But it does not provide any information on whether a fragment has swallowed several nucleons or not. To check this, we use a quantity called the "Gain" [25]. The Gain represents the percentage of nucleons that a fragment has swallowed between two consecutive time steps. Let $\mathrm{N}_{\alpha}^{f}$ be the number of nucleons belonging to a fragment $\alpha$ at time $t$. Let $\mathrm{N}_{\alpha \beta}^{f}$ be the number of nucleons

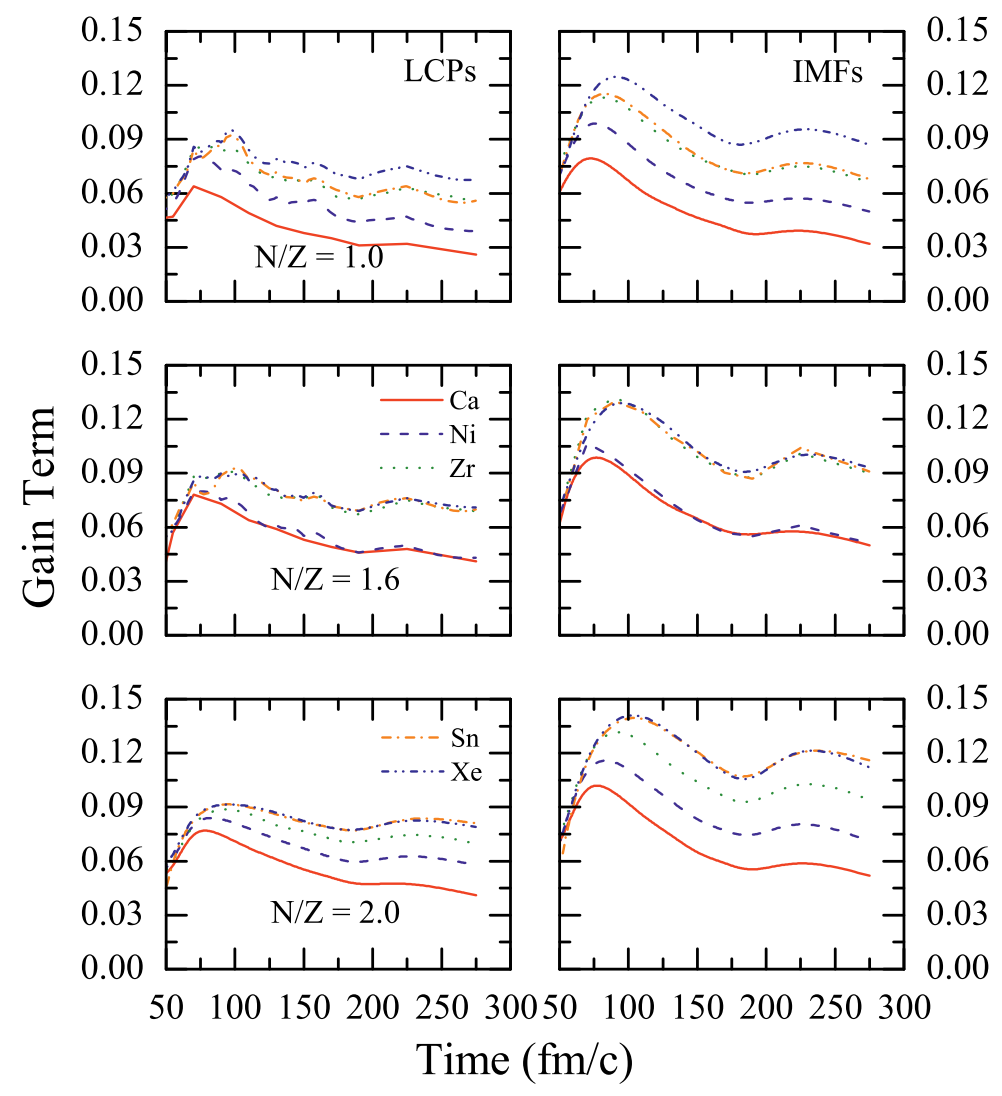

Figure 2. (Color Online) Gain term as a function of time for LCPs (left panels) and IMFs (right panels). The lines have the same meaning as in Fig. 1. 
which were in cluster $\alpha$ at time $t$ and are in cluster $\beta$ at time $t+\Delta t$. The Gain term is defined as:

$$
\operatorname{Gain}(t+\Delta t / 2)=\sum_{\alpha} \eta \times \frac{\sum_{\beta}\left(N_{\beta}^{f}-N_{\alpha \beta}^{f}\right)}{N_{\alpha}^{f}} ;
$$

$\eta=0.0,0.5$, and 1.0 if $\mathrm{N}_{\alpha \beta}^{f}<0.5 \mathrm{~N}_{\beta}^{f}, \mathrm{~N}_{\alpha \beta}^{f}=0.5 \mathrm{~N}_{\beta}^{f}$ and $\mathrm{N}_{\alpha \beta}^{f}>0.5 \mathrm{~N}_{\beta}^{f}$, respectively. Naturally, a true Gain for a fragment $\alpha$ occurs only if its nucleons constitute at least half of the mass of the new fragment $\beta$. The Gain term will tell us whether the interactions among fragments have ceased or not.

In Fig. 2, we display the gain term for LCPs and IMFs. As discussed earlier, the value of the persistence coefficient is slightly higher in the case of LCPs. Therefore, the gain term will be smaller for LCPs as shown in Fig. 2. As evident from the figure, in the case of heavier systems, the gain term has a higher value because of the larger number of nucleon-nucleon interactions.

The rapidity distribution is assumed to give information about the degree of thermalization achieved in heavy-ion reactions. The rapidity distribution of the $i^{\text {th }}$ particle is defined as [3]:

$$
Y(i)=\frac{1}{2} \ln \frac{\mathbf{E}(i)+\mathbf{p}_{z}(i)}{\mathbf{E}(i)-\mathbf{p}_{z}(i)},
$$
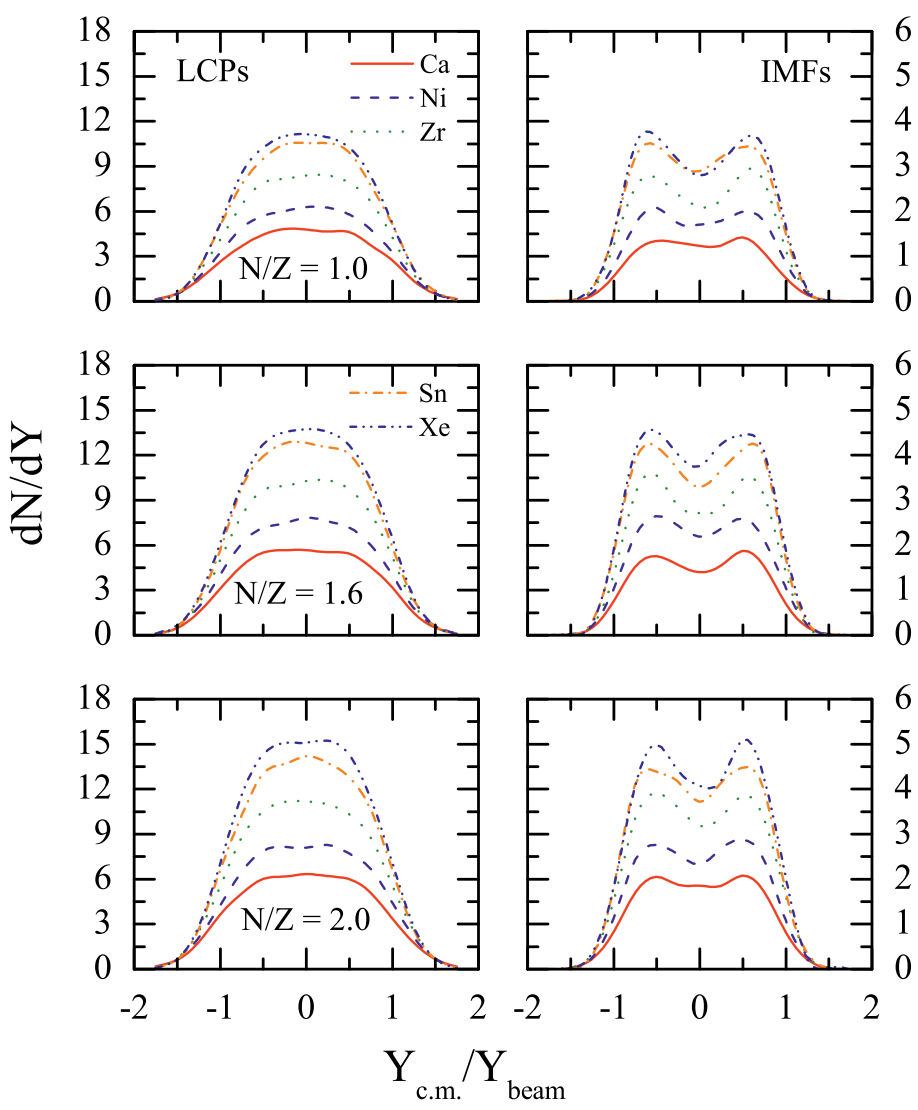

Figure 3. (Color Online) The rapidity distribution, $\mathrm{dN} / \mathrm{dY}$, as a function of reduced rapidity, $\mathrm{Y}_{c . m .} / \mathrm{Y}_{\text {beam }}$. The lines have the same meaning as in Fig. 1. 
Here $\mathbf{E}(i)$ and $\mathbf{p}_{z}(i)$ are, respectively, the total energy and longitudinal momentum of the $i^{\text {th }}$ particle. Naturally, for complete equilibrium, a single Gaussian shaped peak is expected.

In fig. 3, we display the rapidity distribution of LCPs (left panels) and IMFs (right panels). We observed nearly a single Gaussian shape for LCPs in the case of heavy colliding nuclei, whereas the sharp peak flattens for lighter colliding nuclei. Whereas IMFs exhibit a peak at the target/projectile rapidity, indicating a non-equilibrium situation. Thus, these originate from the surface of colliding nuclei.

The quantities which are closely related to the degree of thermalization are the relative momentum $\left\langle K_{R}\right\rangle$ and the anisotropy ratio $\left\langle R_{a}\right\rangle$. The average relative momentum of two colliding fermi spheres is defined as $[3,18,26]$ :

$$
\left\langle K_{R}\right\rangle=\left\langle\left|P_{P}(\mathbf{r}, t)-P_{T}(\mathbf{r}, t)\right| / \hbar\right\rangle,
$$

where

$$
P_{k}(\mathbf{r}, t)=\frac{\sum_{j=1}^{A_{k}} P_{j}(t) \rho_{j}(\mathbf{r}, t)}{\rho_{k}(\mathbf{r}, t)} .
$$

Here $\mathrm{P}_{j}$ and $\rho_{j}$ are the momentum and density experienced by the $j^{\text {th }}$ particle and $k$ stands for either target or projectile and $\mathbf{r}$ refers to a space point in a central sphere of $2 \mathrm{fm}$ radius in which all calcula-

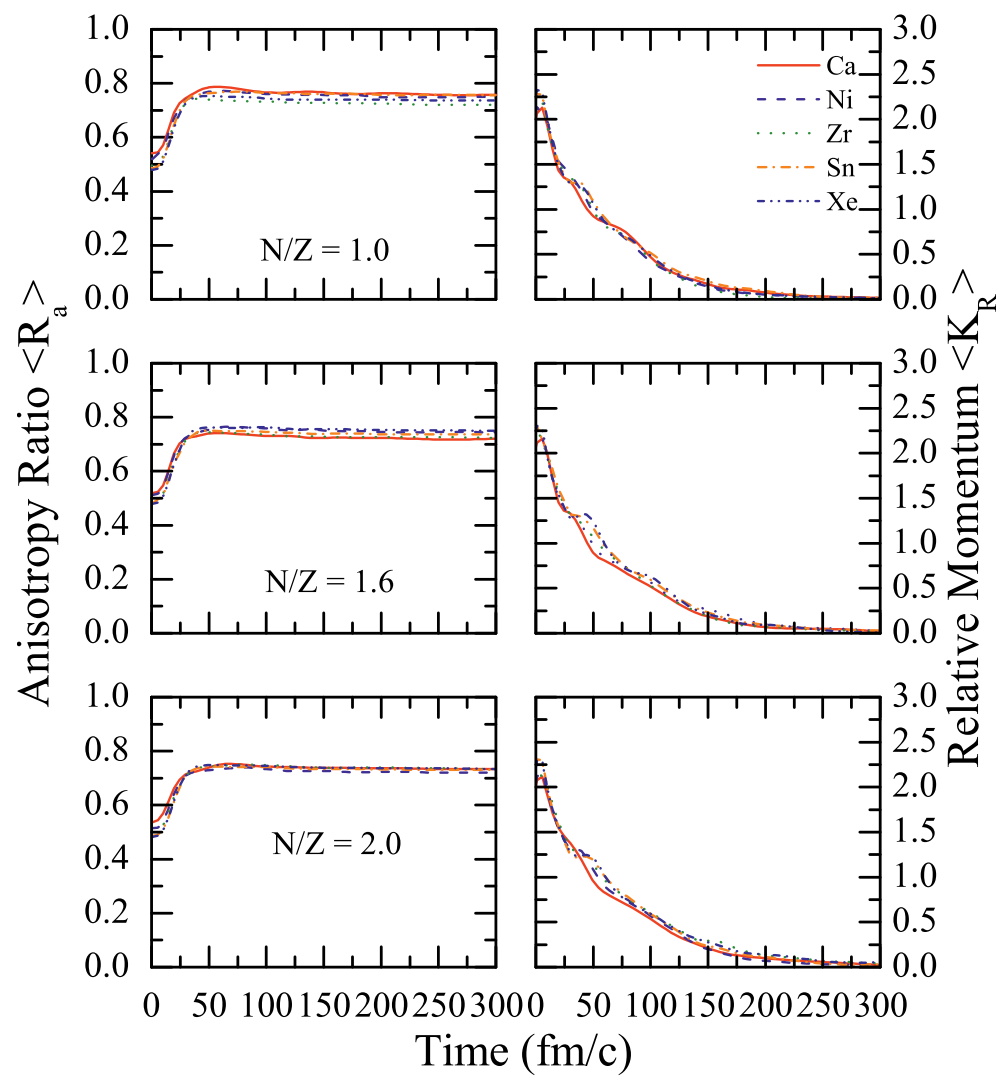

Figure 4. (Color Online) The time evolution of the anisotropy ratio (left panels) and relative momentum (right panels). The lines have the same meaning as in Fig. 1 
tions are made. The $\left\langle K_{R}\right\rangle$ is an indicator of local equilibrium because it depends on the local position $r$.

The second quantity is the anisotropy ratio which is defined as $[3,18,26]$ :

$$
\left\langle R_{a}\right\rangle=\frac{\sqrt{\left\langle p_{x}^{2}\right\rangle}+\sqrt{\left\langle p_{y}^{2}\right\rangle}}{2 \sqrt{\left\langle p_{z}^{2}\right\rangle}} .
$$

The anisotropy ratio $\left\langle R_{a}\right\rangle$ is an indicator of the global equilibrium of the system because it represents the equilibrium of the whole system and does not depend upon the local positions. The full global equilibrium averaged over a large number of events will correspond to $\left\langle R_{a}\right\rangle=1$.

In Fig. 4, we display the $\left\langle R_{a}\right\rangle$ ratio (left panels) and $\left\langle K_{R}\right\rangle$ (right panels) as a function of time for different system masses at $\mathrm{N} / \mathrm{Z}=1.0,1.6$ and 2.0. It is clear from the figure that the anisotropy ratio changes to a greater extent during the high density phase. Once the high density phase is over, no more changes occur in the thermalization. It is interesting to see that the relative momentum is large at the start of the reaction, and finally at the end of the reaction, the value of $\left\langle K_{R}\right\rangle$ is nearly zero. This means that at the end of the reaction, local equilibrium is nearly reached. However, the saturation time is nearly the same throughout the mass range. The neutron content of a colliding pair has a negligible effect on the thermalization achieved in a reaction.

\section{Summary}

In summary, we studied the stability of the fragments and the thermalization achieved in collisions of neutron-rich colliding pairs at the energies where the maximum production of intermediate mass fragments occurs. The stability of the fragments is checked through the persistence coefficient and gain term and information about the thermalization and stopping is obtained via the relative momentum, anisotropy ratio, and rapidity distribution. We found that the saturation value of the persistence coefficient is slightly higher in the case of LCPs compared to IMFs. Also, the value of the persistence coefficient is lower in the case of heavier colliding nuclei compared to lighter ones due to ongoing interactions among the nucleons. In the case of the anisotropy ratio and relative momentum, nearly mass independent behavior is observed. Our findings showed a weak dependence of these quantities on the neutron content of the colliding pair.

\section{Acknowledgements}

This work is supported by the Department of Science and Technology (DST), Government of India. The author is thankful to Professor Rajeev K. Puri for enlightening discussions on the present work.

\section{References}

[1] P. B. Gossiaux and J. Aichelin, Phys. Rev. C 56, 2109 (1997).

[2] M. B. Tsang et al., Phys. Rev. Lett. 71, 1502 (1993).

[3] J. K. Dhawan et al., Phys. Rev. C 74, 057901 (2006).

[4] Y. K. Vermani S. Goyal and R. K. Puri, Phys. Rev. C 79, 064613 (2009).

[5] A. D. Sood and S. Kaur, Pram. J. Phys. 76, 909 (2011).

[6] J. K. Dhawan and R. K. Puri, Phys. Rev. C 74, 054610 (2006). 
[7] R. Ogul et al., Phys. Rev. C 83, 024608 (2011); W. Trautmann et al., Int. J Mod. Phys. E 17, 1838 (2008); C. Sfienti et al., Phys. Rev. Lett. 102, 152701 (2009).

[8] V. Baran et al., Nucl. Phys. A 703, 603 (2002); F. Gagnon-Moisan et al., Phys. Rev. C 86, 044617 (2012).

[9] G. J. Kunde et al., Phys. Rev. Lett. 77, 2897 (1996).

[10] F. S. Zhang et al., Phys. Rev. C 60, 064604 (1999).

[11] R. Płaneta et al., Phys. Rev. C 77, 014610 (2008).

[12] S. Kaur and R. K. Puri, Phys. Rev. C 87, 014620 (2013).

[13] S. Kumar and Y. G. Ma, Nucl. Phys. A 898, 59 (2013); Rajni, S. Kumar, and R. K. Puri, Nucl. Phys. A 875, 173 (2012).

[14] Rajni, S. Kumar, and R. K. Puri, Nucl. Phys. A 875, 173 (2012).

[15] B. A. Li, Z. Ren, C. M. Ko and S. J. Yennello, Phys. Rev. Lett. 76, 4492 (1996); R. Pak et al. Phys. Rev. Lett. 78, 1022 (1997); ibid., 78, 1026 (1997).

[16] S. Gautam et al., J. Phys. G: Nucl. Part. Phys. 37, 085102 (2010); S. Gautam et al., Phys. Rev. C 83, 034606 (2011); ibid., 83, 014603 (2011).

[17] K. S. Vinayak and S. Kumar, Eur. Phys. J. A 47, 144 (2011); S. Kumar, S. Kumar, R.K. Puri, Phys. Rev. C 81, 014611 (2010); V. Kaur, S. Kumar, and R. K. Puri, Phys. Lett. B 697, 512 (2011).

[18] S. Gautam, Phys. Rev. C 83, 064604 (2011); S. Gautam and R. K. Puri, Phys. Rev. C 85, 067601 (2012).

[19] C. Hartnack et al., Eur. Phys. J. A 1, 151 (1998).

[20] J. Aichelin, Phys. Rep. 202, 233 (1991).

[21] S. Goyal and R. K. Puri, Phys. Rev. C 83, 047601 (2011).

[22] Y. K. Vermani and R. K. Puri, Nucl. Phys. A 847, 243 (2011); Y. K. Vermani and R. K. Puri, J. Phys. G: Nucl. Part. Phys. 36, 105103 (2009); Y. K Vermani, J. K Dhawan, S. Goyal, R. K. Puri, and J. Aichelin, J. Phys. G: Nucl. Part. Phys. 37, 015105 (2010); Y. K. Vermani and R. K. Puri, Euro. Phys. Lett. 85, 62001 (2009).

[23] R. Bansal, S. Gautam, R. K. Puri, and J. Aichelin, Phys. Rev. C 87 (2013) 061602(R).

[24] R. K. Puri and J. Aichelin, J. Comp. Phys. 162, 245 (2000); R. K. Puri et al., Phys. Rev. C 54, R28 (1996); P. B. Gossiaux et al., Nucl. Phys. A 619, 379 (1997).

[25] R. K. Puri et al., Pram. J. Phys. 59, 19 (2002).

[26] D. T. Khoa, N. Ohtsuka, M. A. Matin, A. Faessler, S. W. Huang, E. Lehmann, and R. K. Puri, Nucl. Phys. A 548, 102 (1992); R. K. Puri, N.Ohtsuka, E. Lehmann, Amand Faessler, M. A. Matin, D. T. Khoa, G. Batko, and S. W. Huang, ibid., 575, 733 (1994). 